\title{
Development of infrared single mode fibers for 2 wavelength bands of the DARWIN mission: Test results of prototypes
}

\author{
Lun K. Cheng ${ }^{* a}$, Niels Dijkhuizen ${ }^{\mathrm{a}}$, Remco Nieuwland ${ }^{\mathrm{a}}$, Anne-Jans Faber ${ }^{\mathrm{a}}$, Wim Gielesen ${ }^{\mathrm{a}}$, Jacques \\ Lucas $^{\mathrm{b}}$, Catherine Boussard-Plédel ${ }^{\mathrm{b}}$, Patrick Houizot ${ }^{\mathrm{b}}$, João Pereira Do Carmo ${ }^{\mathrm{c}}$ \\ ${ }^{a}$ TNO Science and Industry, P.O. Box 155, 2600 AD Delft, The Netherlands; \\ ${ }^{\mathrm{b}}$ University of Rennes, Glass and Ceramic Laboratory, Campus of Beaulieu, 35042 Rennes, Frans; \\ ${ }^{c}$ European Space Agency, P.O. Box 299, 2200 AG Noordwijk ZH, The Netherlands
}

\begin{abstract}
Various space telescope array systems are being considered to investigate other terrestrial planets orbiting around nearby stars in order to find extra-terrestrial life. One of them is the DARWIN mission of the European Space Agency (ESA). The required technology is the nulling interferometer. The challenge of nulling is making the null in the interferometric signal sufficiently deep to cancel the light from the bright star during the collection of light from its surrounding planets. The performance of the nulling is limited by the wavefront quality of the beams. The wavefront error can be reduced by filtering using a single mode fiber. For the DARWIN mission, the operational wavelength range is $6.5-20 \mu \mathrm{m}$. Within the current ESA project, this is covered by a dual-band fiber system. A chalcogenide glass fiber based on the Te-As-Se (TAS) composition is selected to be used for the short wavelength band. For the long wavelength band up to $20 \mu \mathrm{m}$, Tellurium based glass is proposed. Different samples of various composition based on Te glass are manufactured and tested. The fibers are designed by TNO and different prototypes have been manufactured by the University of Rennes. Test setups are developed to demonstrate/investigate the single mode operation. Cladding modes are found to disturb the single mode operation. The effect of cladding modes is modeled. Solutions to eliminate the cladding modes are investigated and tested.
\end{abstract}

Keywords: fiber optic, interferometer, interferometry, astrometry, single mode

\section{INTRODUCTION}

One of Darwin's two main objectives is to find other terrestrial planets orbiting around nearby stars. This can be done by performing a simple spectroscopic analysis of their atmosphere. This spectroscopic analysis could show that some chemical components usually associated with life are present in some exoplanets and therefore there is a possibility for extraterrestrial life. The star/planet systems of interest are those with stars having properties similar to our Sun and with planets located at a distance from the star in a way similar to our Earth in the so-called habitable zone. A telescope capable to separate these two objects at a wavelength of five $\mu \mathrm{m}$ will require a diameter of $50 \mathrm{~m}$. A smaller diameter could be sufficient at visible wavelengths. However, in the visible spectrum, the signal from the planet is much smaller with respect to the star signal than in the thermal infrared. This is because in the visible spectrum, the planet only reflects a part of the light emitted by the star, whereas in the thermal infrared it has a proper thermal emission. This imposes the choice of the long wavelengths and thus the need for large telescopes.

A monolithic telescope with a $50 \mathrm{~m}$ diameter is presently not considered as a feasible option. This led to the proposal to use isolated telescopes separated by a $50 \mathrm{~m}$ distance instead to remain within the development of today's technological possibilities. Such a system only gives information at one angular frequency in the order of $\lambda / B$, where $B$ is the distance separating the telescopes. By changing this baseline B it is thus possible to cover all possible resolution values between zero and the maximum available value.

A vital function of Darwin is the so-called "nulling" operation. Even at infrared wavelengths, the flux emitted by the star is extremely large compared to the flux emitted by the planet. Without nulling, the star signal would completely mask the planet signal. The principle of nulling is based on adding a phase difference of $\pi$ radians in one of the two beams before recombining them in the focal plane so that they interfere destructively at zero optical path difference. The signal from a point like star on the optical axis of the constellation is thus theoretically null in the focal plane. The planet signal

Photonic Fiber and Crystal Devices: Advances in Materials and Innovations in Device Applications III, edited by Shizhuo Yin, Ruyan Guo, Proc. of SPIE Vol. 7420, 742006 - (c) 2009 SPIE · CCC code: 0277-786X/09/\$18 · doi: 10.1117/12.825251 
is not "nulled" because the planet is slightly off-axis so that the phase between the various beams is different from that of the star. This trick reduces the contrast between star and planet and enables the detection of the planet. The challenge of nulling is making the null sufficiently deep. The following requirements have also to be met to achieve a sufficient nulling:

- Exact phase shift difference of $\pi$ radians between the beams.

- The phase between the beams must be extremely stable to ensure a good nulling performance during the exposure, which can be up to several tens of hours.

- Matching of polarisation of the beams.

- The amplitude of the beams must be the same as well as the repartition of this amplitude on the pupil so that all flux from the telescopes fully interferes.

- Nearly perfect wavefront of the beams.

The latter can be realised by wavefront error filtering before the detection. The preferable concept for the wavefront filter is a single mode waveguide [1]. For the application of the single mode waveguide as wavefront filter in Darwin, the waveguide has to meet the operational conditions in space like the operational temperature and the radiation susceptibility. Furthermore, a minimal insertion loss is required. The objective of the current project is to develop single mode waveguides to be used as wavefront filter in the Darwin infrared spectral region (6.5 to $20 \mu \mathrm{m})$. This range covers the characteristic spectral lines for Earth and other planets.

\section{DUAL WAVELENGTH BANDS CONFIGURATION}

For optical system in astronomy, the transmission is of major importance. The transmission of a fiber optic system includes the attenuation of the fiber material and the coupling efficiency. The coupling efficiency into a fiber is determined by the overlapping integral between the Airy spot and the mode profile. The maximum coupling efficiency $\eta_{\max }$ is found to be $79 \%$ for the cut-off wavelength. For a conventional optical system, the dimension of the Airy spot is proportional to the wavelength while the Mode Field Diameter (MFD) of the fundamental mode in a step index fiber has an exponential-like relation with the wavelength. Therefore, the coupling efficiency $\eta$ will depend on the wavelength. We introduce the normalised coupling efficiency as $\eta / \eta_{\max }$. For a fibre of which the coupling efficiency is optimised for the cut-off wavelength $\lambda_{\mathrm{c}}$, the normalised coupling efficiency will drop with longer wavelengths (Fig. 1).

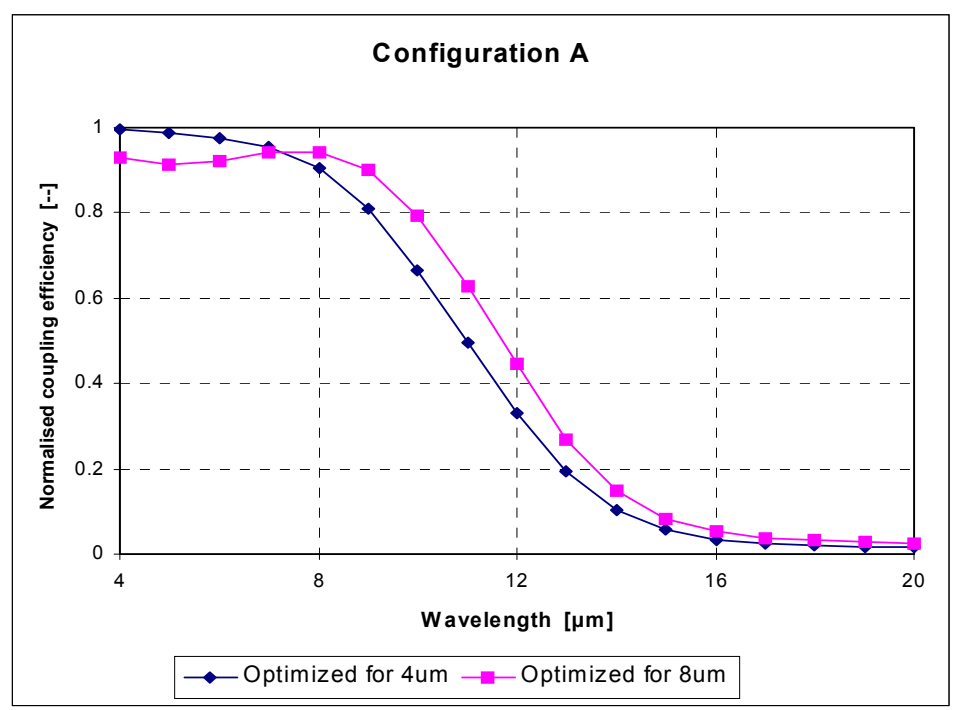

Fig.1 Normalised coupling efficiency as function of the wavelength for a fibre with $\lambda_{\mathrm{c}}=4 \mu \mathrm{m}$. Impact of optimising the normalised coupling efficiency for different wavelength is shown.

This will limit broadband operation of the single mode fibre for wavefront filtering and splitting the entire wavelength range into several bands will be necessary. Optimising the light coupling for a wavelength longer than $\lambda_{\mathrm{c}}$ by adapting 
the focusing optics for the input beam will reduce the maximum normalised coupling efficiency slightly. However, the normalised coupling efficiency will remain high for a large wavelength range. This is shown in Fig. 1. When the coupling into the fibre with $\lambda_{\mathrm{c}}=4 \mu \mathrm{m}$ is optimised for $8 \mu \mathrm{m}$, the normalised coupling efficiency is about $90 \%$ from 4 $\mu \mathrm{m}$ to about $9 \mu \mathrm{m}$. This allows broad wavelength band operation of the single mode fiber for wavefront-filtering and limits the required number of wavelength bands. In the previous Darwin SMW project with required operational wavelength range of 4 to $20 \mu \mathrm{m}$, a dual band system with a short wavelength (SW) fiber and a long wavelength (LW) fiber is proposed. By designing the input beams for optimising the coupling efficiency of SW fiber for about $7.5 \mu \mathrm{m}$ and LW fiber for about $18 \mu \mathrm{m}$, a system with a coupling efficiency higher than $80 \%$ for the entire wavelength range from 4 to $20 \mu \mathrm{m}$ is demonstrated.

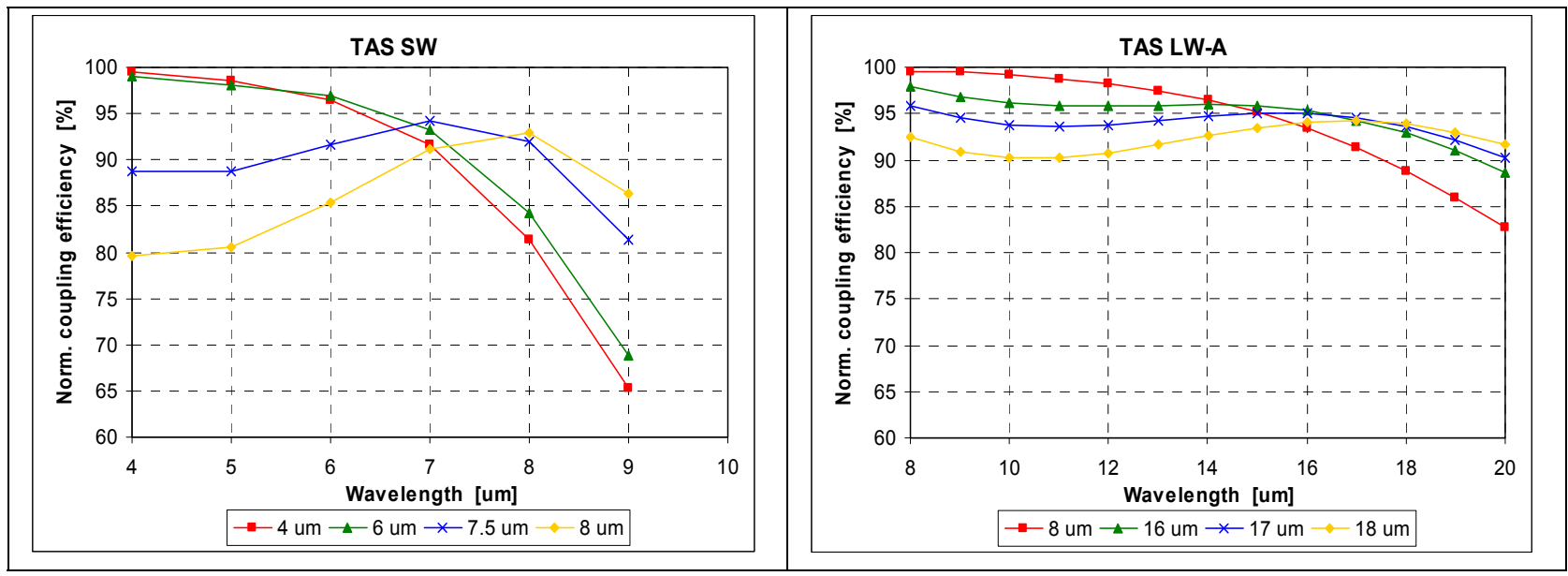

Fig. 2 Normalised coupling efficiency of a dual band system as a function of the wavelength. Left: SW fiber for the short wavelength band; right: LW fiber for the long wavelength band.

As the wavelength range of the current Darwin SMW project is reduced to $6.5-20 \mu \mathrm{m}$, the dual band configuration is expected to be suitable. The wavelength range will be divided into the short wavelength range $(6.5->12 \mathrm{um})$ and the long wavelength range ( $<12$ um to $20 \mathrm{um})$.

To cover the DARWIN wavelength range from $6.5 \mu \mathrm{m}$ to $20 \mu \mathrm{m}$, material with sufficient high transmission has to be found. Solids with IR transmitting properties include both certain crystalline materials and specific glass types (amorphous materials). Relevant crystalline materials include halides (e.g. $\mathrm{KBr}, \mathrm{KCl}, \mathrm{KI}$ ), chalcogenides (e.g. $\mathrm{ZnS}$, $\mathrm{ZnSe}$ ) and semiconductors (e.g. Ge, GaAs, diamond). Relevant glass types include multi-component fluoride glasses, halide glasses and chalcogenide glasses (sulphides, selenides and tellurides). The main advantage of using glasses over crystalline materials is that, generally, glasses can be drawn into fibres with any required geometry for single mode waveguides. Since glasses are amorphous materials, no crystal boundaries are present, as in the case of polycrystalline materials, which cause scatter losses of propagating light signals and deteriorate the wavefront-filter properties of single mode fibres. The most stable glasses with transmission up to about $20 \mu \mathrm{m}$ are chalcogenide glasses.

In previous studies for ESA [2, 3], the Te-As-Se (TAS) glasses are identified to be suitable for the spectral region 6.5 $12 \mu \mathrm{m}$ while Tellurium-based glasses with the heaviest Te as main chalcogen is regarded to be a good candidate for $12-$ $20 \mu \mathrm{m}$. The Te-glass development is less mature. Therefore, extra attention has to be paid to the Te-glass based single mode fiber for the long wavelength band.

\subsection{Te glass development for the long wavelength fiber}

High Tellurium (Te) content chalcogenide glass fibers are considered as candidates for single mode waveguides in the upper wavelength range $(10-20 \mu \mathrm{m})$ of the DARWIN mission. The main problem of high Te content glass is 
crystallization. The possibility of drawing high Te content fiber has to be demonstrated. Different mono index high Te content fibers were manufactured from the following chemical glass compositions:

\section{- TeGeGal \\ - TeGeI \\ - TeGeSe}

Using special raw materials preparation, chemical purification and glass melting procedures [4], glass rods of about 9 $\mathrm{mm}$ diameter and about $10 \mathrm{~cm}$ length were manufactured. These glass rods were drawn to mono index fibers (ø from $400-550 \mu \mathrm{m}$ ), using a special fiber drawing machine [5]. Several meters of three TeGeGaI type mono-index fibers (two non-purified $\varnothing 400 \mu \mathrm{m}$ and one purified $\varnothing 400 \mu \mathrm{m} / \varnothing 520 \mu \mathrm{m}$ ) were drawn, showing a good surface quality, free of defects or crystallization (see Fig. 3). Similar preparation procedures were used to manufacture fibers of more than 10 meters length from the TeGeI and TeGeSe glass compositions. The TeGeGaI fiber is found to have the best manufacturability and is selected for future development of the LW fiber.
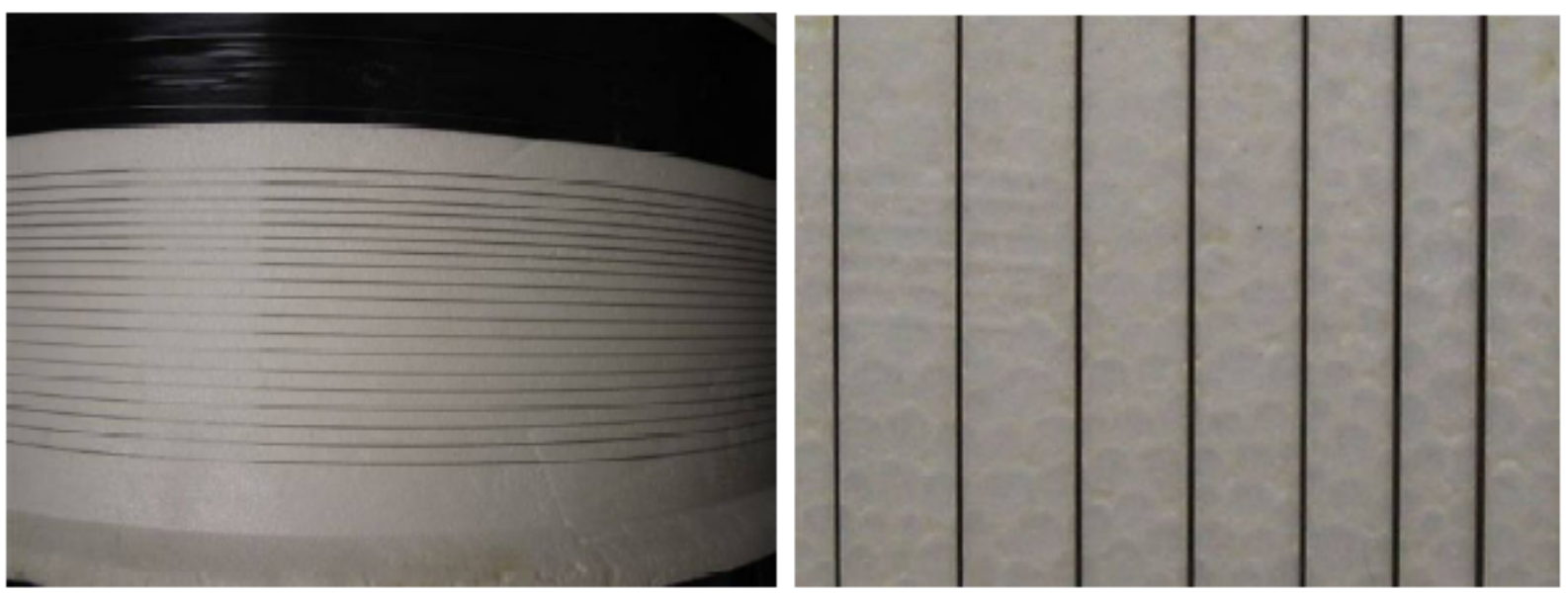

Fig. 3 Mono-index TeGeGaI fiber on a drum.

Since high Te-glasses are semiconducting materials, the optical properties of Te-glass fibers strongly depend on temperature. In order to simulate the impact of temperature in space, a qualitative experiment was carried out on a 135 $\mathrm{mm}$ long TeGeGaI-fiber, cooled by liquid nitrogen (LN2), using the broadband FTIR set-up. The transmission spectrum measured at low temperature was compared to the spectrum of the same fiber at room temperature. At LN2 temperature, the attenuation is found to be lower. The improvement is shown in Fig. 4.

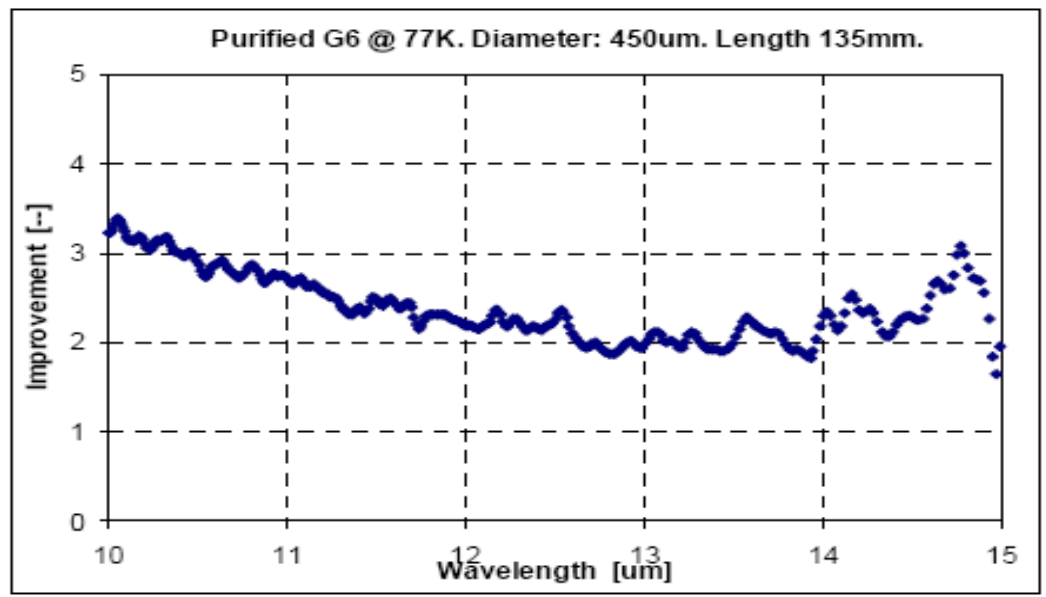

Fig. 4 Factor of improvement in transmission of a TeGeGaI fiber at liquid nitrogen compared to room temperature. 


\section{Prototype Te-glass LW fiber}

The first prototype Te-glass step index fiber based on TeGeGaI glasses is manufactured by multi-steps rod-in-tube method and no crystallization is observed visually. The attenuation is measured to be $0.2 \mathrm{~dB} / \mathrm{cm} @ 10.6 \mu \mathrm{m}$ and room temperature. The refractive index of the Te glass will be measured in the near future. The results will be used for the design of the LW fiber.

\subsection{TAS glass development for the short wavelength fiber}

The refractive index of the TAS glass is measured in the previous Darwin SMW project. This is used for the design of the short wavelength fiber:

- Core radius

- Core material

- Cladding thickness

- Cladding material

- Calculated cut-off wavelength

- NA
: 17.5 um

: TAS composition X (refractive index=2.909@10.6 $\mu \mathrm{m})$

: 250 um

: TAS composition Y (refractive index =2.906@10.6 $\mu \mathrm{m})$

$: 5.7$ um

$: 0.13$

\section{Prototypes TAS glass SW fiber}

3 prototypes of the TAS glass SW fibers are manufactured. The attenuation measured by cut-back method is found to be between 0.08 and $0.11 \mathrm{~dB} / \mathrm{cm} @ 10.6 \mu \mathrm{m}$ and room temperature. These fibers are used for the investigation of absorption coating. The results are discussed in Section 3.2.

\section{CLADDING MODE SUPPRESSION}

To ensure a sufficient nulling depth, the amount of optical power in the higher order mode at the output of the singlemode fiber has to be limited. For the Darwin SMW, the requirement for the higher order mode suppression $\boldsymbol{A}$ is $10^{4}$ with a goal of $10^{6}$.

A single mode fiber has a core-cladding structure and mostly includes a coating. The losses of the leaky higher-order modes depend on both the thickness of the cladding and the refractive index of the coating material and can be maximised via careful choice of these parameters. However, for short device lengths and where a high quality single mode output is required (such as for the case of the wavefront filter considered here) the fraction of power carried in the leaky $\mathrm{LP}_{11}$-like mode can still be significant and must be taken into account when determining the minimum device length. Similarly, the fraction of power carried by the cladding modes can also have a significant impact on the filtering capabilities. This is a particularly important consideration in high index materials such as those required for the Darwin wavelength range, since the cladding itself forms a large multimode waveguide with a high NA in the case of the uncoated fibre. Coating the fibre with an absorbing material allows these cladding modes to be suppressed. In previous studies, absorbing gallium-layers have been applied manually and is found to be able to suppress the higher order modes (HOM) significantly. However, Ga is difficult to handle due to the low melting point. Even at room temperature, Ga becomes soft and mechanically unstable. In order to find other absorption coating material with better manufacturability (and absorption), cladding mode suppression in the TAS SW fiber by different coating materials is modelled. The imaginary part of the refractive index $(k)$ of a material is responsible for the absorption. For the modelling the effect of $\mathrm{Cr}(k \approx 15 @ 10.6 \mu \mathrm{m})$ on the HOMs is compared to that of Ga $(k \approx 30 @ 10.6 \mu \mathrm{m})$. Furthermore, different TAS SW fibers are manufacturer using the multi-steps rod-in tube method and the effect of different coating materials are measured.

\subsection{Modeling of cladding modes}

The numerical modeling is performed using a commercial software package named COMSOL Multiphysics ${ }^{\mathrm{TM}}$ that is based on the Finite Element Method (FEM). This technique can be used to provide an accurate vector analysis of the modal properties of waveguides with arbitrary refractive index profiles. In the FEM, the transverse refractive index 
profile is split into distinct homogeneous subspaces. This involves dividing the index profile into a mesh of triangles (the finite elements). The size of the triangles as well as the density of the mesh (of triangular finite elements) depends on the dimensions of the different layers of the fiber and the localization of the modal field. A layer that has a smaller thickness requires smaller triangles for the mesh while a finer mesh is required in regions where the optical field intensity is expected to be high. Graded mesh density reduces the calculation time while maintaining the accuracy. Once the mesh has been formed, the classical Maxwell differential equations are solved for each of the elementary subspaces, taking into account the continuity conditions of the electric and magnetic fields. This method can be used to evaluate the complex propagation constants (effective index and loss) of the modes of finite lossy structures.

The typical number of cladding modes supported by the DARWIN fiber is $>1000$. Out of these, around 300-400 modes have an effective refractive index near the cladding refractive index and have low losses. These are the cladding modes that have been solved in the program. The following analyses of the model properties of the fiber are performed:

- Confinement loss of the modes $(\mathrm{dB} / \mathrm{m})$

- Overlap with Airy disk (\%). This determines the coupling efficiency of the particular mode.

- Fractional optical power in the core (\%).

The combination of the confinement loss and the coupling efficiency determines the optical power of the particular mode at the end of the fiber. Using these results, the transmission of the fundamental mode and $\boldsymbol{A}$, the suppression of the higher order modes can be simulated. The latter determines the required length of the SMW to achieve $\boldsymbol{A}=10^{6}$.

An un-infinite cladding will generate cladding modes. It is observed that the $\mathrm{LP}_{11}$ mode does not automatically have the lowest confinement loss amongst all the higher order modes. Thus, in order to obtain a realistic estimate of the required fiber length for sufficient higher order mode suppression, it is important to take into account the coupling efficiencies of the various cladding modes as well as their respective confinement losses. However, the number of cladding mode for the current fiber design is $\sim 1000$ and many of these modes have low losses $(<0.01 \mathrm{~dB} / \mathrm{m})$. Furthermore, the modes that have the lowest losses may have a very small overlap with the Airy disk whereas some modes that have a significant overlap may not have very low losses. Therefore, criteria need to be defined for considering only those modes that would significantly affect the performance of the fiber. A high overlap with the Airy disk is found to be the best criteria $[6]$.

\section{Modeling results of TAS SW fiber with $600 \mathrm{~nm}$ Cr coating:}

Table 1 Specifications of the HOMs that have overlap of $>0.1 \%$ with the Airy intensity distribution. The required length for $10^{-6}$ suppression is shown in the last column. Profile of the 2 modi with highest overlap is also shown.

HOMs that have overlap $>0.1 \%$ with input field

\begin{tabular}{|c|c|c|c|c|c|}
\hline $\begin{array}{c}\text { Effective } \\
\text { index }\end{array}$ & $\begin{array}{c}\text { Overlap } \\
(\%)\end{array}$ & $\begin{array}{c}\text { Confinement } \\
\text { loss }(\mathrm{dB} / \mathrm{m})\end{array}$ & $\begin{array}{c}\text { Fractional } \\
\text { power in } \\
\text { core }(\%)\end{array}$ & $\begin{array}{c}\text { Angle with } \\
\text { fiber axis } \\
\text { (deg.) }\end{array}$ & $\begin{array}{c}\text { Zmin (m) } \\
\left(\mathrm{A}=10^{8}\right)\end{array}$ \\
\hline 2.9126005 & 0.17 & 38.4 & 1.62 & 1.94 & 1.5 \\
\hline 2.9130335 & 0.42 & 46.2 & 1.78 & 1.67 & 1.2 \\
\hline 2.9130339 & 0.34 & 44.9 & 1.78 & 1.67 & 1.3 \\
\hline 2.9132775 & 0.12 & 80.2 & 0.48 & 1.50 & 0.7 \\
\hline 2.9134091 & 0.91 & 44.9 & 1.72 & 1.39 & 1.3 \\
\hline 2.9134093 & 0.53 & 49.1 & 1.71 & 1.39 & 1.1 \\
\hline 2.9137218 & 0.71 & 34.3 & 1.41 & 1.11 & 1.6 \\
\hline 2.9137223 & 0.72 & 36.8 & 1.41 & 1.11 & 1.5 \\
\hline 2.9139658 & 0.70 & 20.4 & 0.97 & 0.83 & 2.8 \\
\hline 2.9139656 & 0.36 & 21.6 & 0.97 & 0.83 & 2.6 \\
\hline 2.9141390 & 0.37 & 8.9 & 0.51 & 0.55 & 6.3 \\
\hline 2.9141390 & 0.18 & 9.6 & 0.51 & 0.55 & 5.9 \\
\hline 2.9142416 & 0.11 & 2.1 & 0.15 & 0.26 & 27.1 \\
\hline
\end{tabular}

Field profile of degenerate pair of HOMs having highest overlap with FM
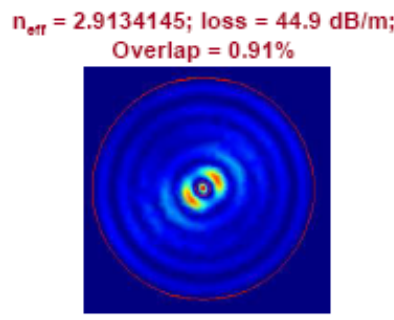

$\mathrm{n}_{\text {eff }}=2.9134142 ;$ loss $=36.8 \mathrm{~dB} / \mathrm{m}$; Overlap $=0.72 \%$

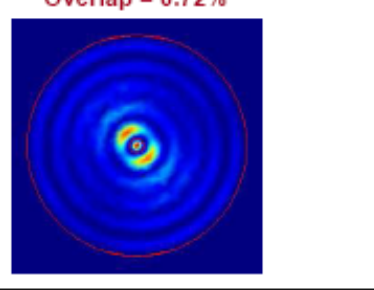


For the TAS SW fiber with $\mathrm{Cr}$ coating, the minimum fiber length $z_{\min }$ to achieve suppression $\mathrm{A}$ of $10^{6}$ is about $27 \mathrm{~m}$. The same calculation is performed for the same TAS SW fiber wit Ga coating. $z_{\min }$ is calculated to be $1.1 \mathrm{~m}$. According to the modelling results, Ga with a higher $k$ than $\mathrm{Cr}$ requires a shorter fiber length to achieve the desired HOM suppression. A survey of materials with a $k$ higher than that of the $\mathrm{Ga}$ is conducted in order to achieve further reduction of the required fiber length. Possible materials are:

- $\mathrm{Au}$

- $\mathrm{Ag}$

- $\mathrm{Al}$

The results are summarized in the table below.

Table 2 Materials for absorption coating.

\begin{tabular}{|c|c|}
\hline & $n+k i @ 10$ um \\
\hline $\mathrm{Au}$ & $8+82 \mathrm{i}$ \\
\hline $\mathrm{Ag}$ & $10+90 \mathrm{i}$ \\
\hline $\mathrm{Al}$ & $35+105 \mathrm{i}$ \\
\hline $\mathrm{Cr}$ & $12+13 \mathrm{i}$ \\
\hline $\mathrm{Ga}$ & $23+32 \mathrm{i}$ \\
\hline
\end{tabular}

\subsection{Absorption coating for stripping of cladding modes}

The effects of $\mathrm{Au}, \mathrm{Ag}$ and $\mathrm{Al}$ for stripping the cladding modes in TAS fibers are compared to that of Ga by inspecting the far field intensity distribution (FFI) @ $10.6 \mu \mathrm{m}$ which is measured by a microbolometer. The metal coatings are applied to different prototype TAS fibers by vacuum deposition under low temperature to avoid thermal degradation of the TAS fiber. The test configuration for the FFI measurement is shown in Fig. 5.

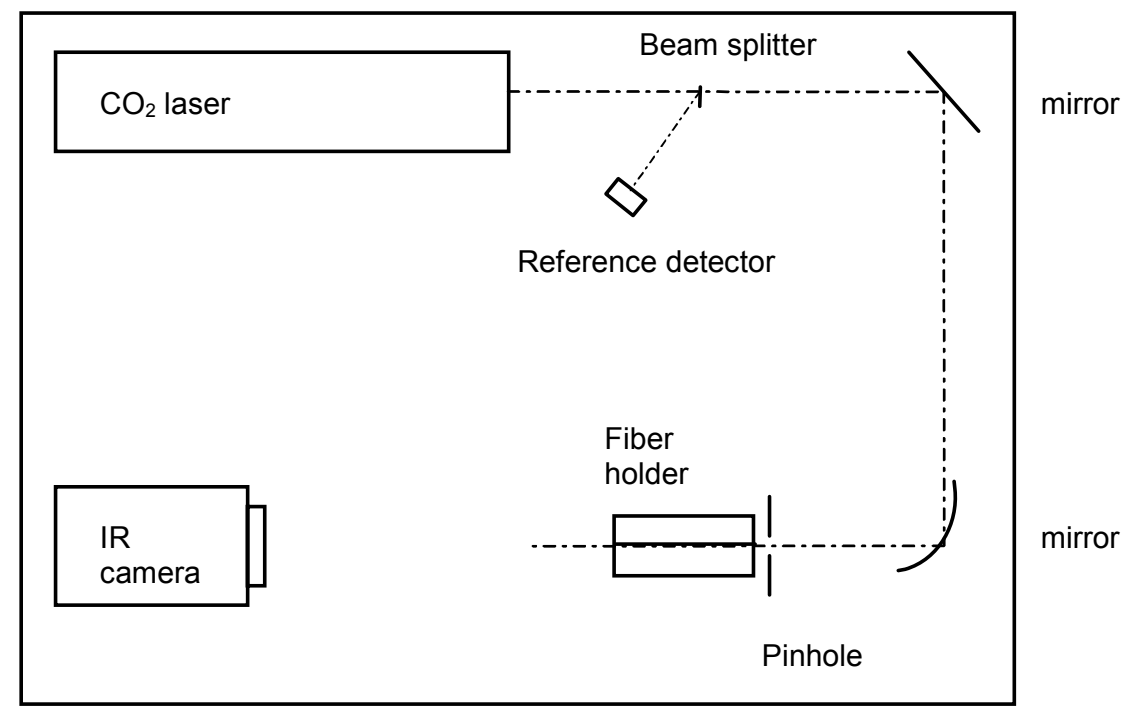

Fig. 5 Test setup for the far field intensity distribution measurement.

All the three alternatives show effect of cladding mode suppression. However, the results are not better than the Ga coating. The FFI measurement result of a $540 \mathrm{~mm}$ Au coated TAS fiber is shown in Fig. 6a. The presence of HOM can clearly be observed. While a $230 \mathrm{~mm}$ Ga coated TAS fiber in the previous Darwin SMW project reveals a Gaussian FFI 
[1]. Apparently, the capability to suppress the HOMs doesn't depend only on the imaginary part of the refractive index of the absorption coating. Further investigation on the absorption coating will be required.

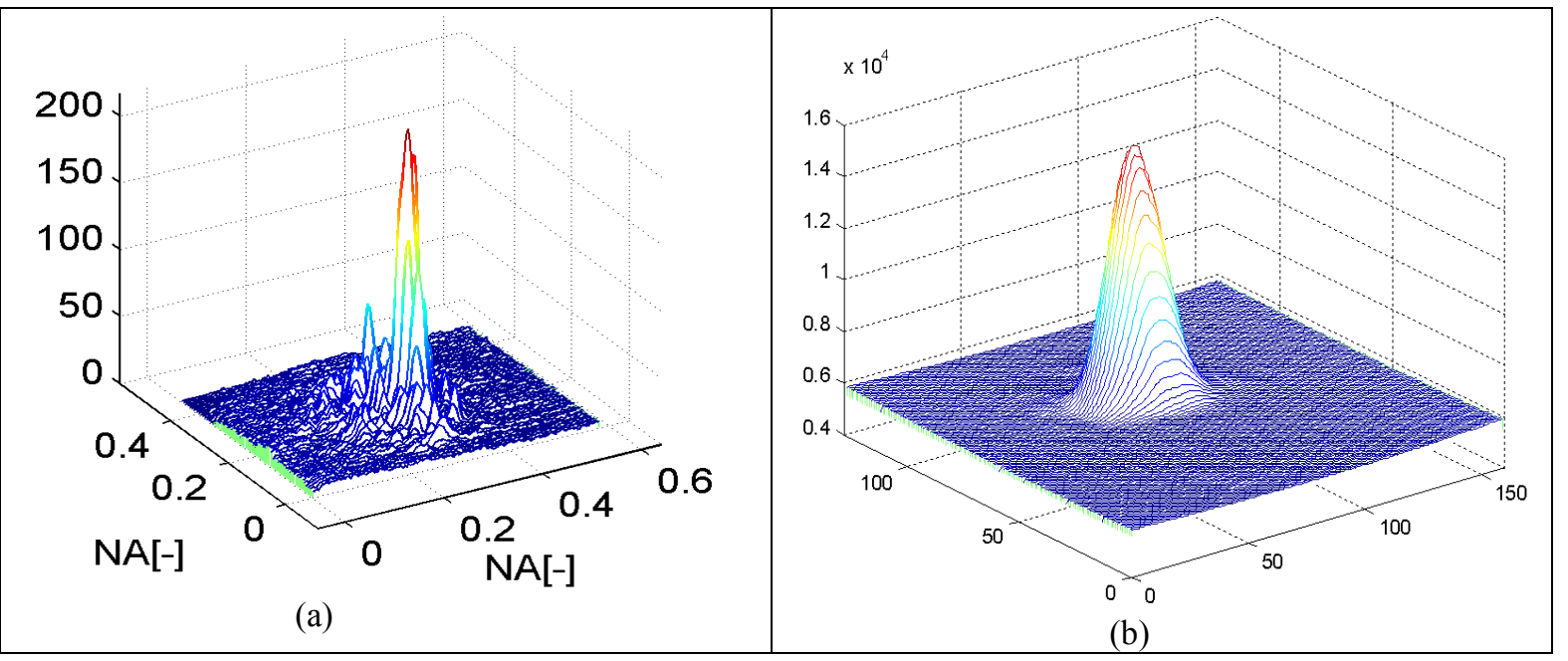

Fig. 6 Comparison between the effects of absorption coating on the far-field intensity distribution. (a) A 540mm TAS fiber with Au coating. (b) A $230 \mathrm{~mm}$ TAS fiber with Ga coating.

\section{CONCLUSIONS}

Design of the dual wavelength bands configuration single mode fiber for wavefront filter in the DARWIN mission is presented. The Te-As-Se (TAS) glasses are identified to be suitable for the spectral region $6.5-12 \mu \mathrm{m}$ while Telluriumbased glasses with the heaviest Te as main chalcogen is regarded to be a good candidate for $12-20 \mu \mathrm{m}$. The possibility of drawing high $\mathrm{Te}$ content fiber has been demonstrated. Different mono index high Te content fibers were manufactured without crystallization. Since high Te-glasses are semiconducting materials, the optical properties of Teglass fibers strongly depend on temperature. Preliminary attenuation measurement down to $\mathrm{LN}_{2}$ temperature showed an improvement of minimum a factor of 2 in the transmission. First prototype Te-glass step index fiber is drawn. No crystallization is observed and the attenuation is measured to be $0.2 \mathrm{~dB} / \mathrm{cm} @ 10.6 \mu \mathrm{m}$ at room temperature. Different prototypes of the TAS SW fiber are manufactured and the measured attenuation varies from 0.08 to $0.11 \mathrm{~dB} / \mathrm{cm} @ 10.6$ $\mu \mathrm{m}$ at room temperature.

The development of the TAS glass based short wavelength range fiber is focused to the cladding mode suppression. The effect of absorption coating is modeled using COMSOL Multiphysics ${ }^{\mathrm{TM}}$. Comparison between $\mathrm{Ga}$ and $\mathrm{Cr}$ as absorption coating material is performed. Ga with a larger imaginary part of the refractive index $(k)$ is calculated to have a stronger suppression of the cladding modes. Different materials with a $k$ larger than $\mathrm{Ga}$ are investigated as absorption coating material for the prototype TAS fibers. The results are not in line with the modeling results. Ga with a lower $k$ is measured to have the best cladding modes suppression capability. Further investigation of the cladding modes suppression mechanism is desired.

\section{ACKNOWLEDGEMENT}

This work is funded by the European Space Agency under contract No.20914/07/NL/CP. The contribution of ORC of Southampton University for the modelling of the higher order modes is acknowledged. 


\section{REFERENCES}

1. L.K. Cheng, A.J. Faber, W. Gielesen, L. Lucas, C. Boussard-Plédel, P. Houizot and J.P. Pereira do Carmo, "Development of broadband infrared single-mode fibers for the DARWIN mission", Proceedings of SPIE, Vol. 6268 (2006).

2. L.K. Cheng, A.J. Faber, W. Gielesen, C. Boussard-Pledel, P. Houizot, J. Lucas and J.P. Do Carmo, "Test results of the infrared single mode fiber for the DARWIN mission", Techniques and Instrumentation for Detection of Exoplanets II, Editor D.R. Coulter, Vol. 5905 (2005).

3. A.J. Faber, L.K. Cheng, W.L.M. Gielesen, C. Boussard-Plédel, S. Maurugeon, B. Bureau, X.H. Zhang, J. Lucas, J. Pereira Do Carmo, "Optical characterization of infrared Telluride class fibers for space use", International Conference on Space Optics (2008).

4. A. Wilhelm, C. Boussard-Plédel, Q. Coulombier, J. Lucas, B. Bureau, P. Lucas, 'Development of farinfraredtransmitting Te based glasses suitable for carbon dioxide detection and space optics', Advanced Materials, Vol. 19, pages 3796-3800 (2007).

5. S. Hocdé, C. Boussard-Plédel, G. Fonteneau, J. Lucas, 'Chalcogen based glasses for IR fiber chemical sensors', Solid State Sciences, 3 (3), papes 279 (2001).

6. S. Dasgupta, N.G.R. Broderick, D.J. Richardson, T. Lewi and A. Katzir, "Improved method for estimating the minimum length of modal filters fabricated for stellar interferometry", OPTICS EXPRESS, Vol. 17, No. 3, pages 1935-1946 (2009). 